\title{
Retroactive inhibition as a function of the locus of categorized response sets
}

\author{
HARRY LEARDO CHIESI \\ University of Pittsburgh, Pittsburgh, Pennsylvania 15260
}

\begin{abstract}
The effects which list-level taxonomic attributes should have upon retroactive inhibition (RI) producing mechanisms in both response-set interference theory (Postman \& Stark, 1969) and Petrich's (1975) retrieval model are discussed. A test of the theories was performed by manipulating the presence (1) or absence (0) of categorized response sets in both original learning (OL) and interpolated learning (IL), and comparing their predictions concerning the relative degree of RI expected in the four conditions $(0-0,0-1$, 1-0,1-1). In line with Petrich's predictions, OL categorization significantly reduced RI, IL categorization significantly increased RI, and 0-1 produced significantly greater RI than 1-0. Moreover, the obtained ranking of the means for the experimental groups in degree of RI $(0-1>0-0>1-0 \cong 1-1)$ was in close agreement with the ranking predicted by Petrich's (1975) model. The results provide support for Petrich's (1975) retrieval model and pose explanatory problems for response-set interference theory (Postman \& Stark, 1969).
\end{abstract}

Petrich (1975) has recently proposed a model for retroactive inhibition (RI) which is based on the assumptions that (a) the first and second lists are stored in separate locations or "memory units," and (b) the intrusion of original learning (OL) responses during interpolated learning (IL) provides "an opportunity to store the intruding first-list responses within the second-list memory unit" (Petrich, 1975, p. 64). According to this retrieval model, the memory unit formed during first-list learning and containing all the first-list pairs becomes inaccessible during second-list learning. This occurs because OL pairs in the OL unit have not been tagged for retrieval during acquisition, while the recently formed IL unit is more closely associated with the experimental context at recall. Under typical experimental conditions, therefore, the number of OL pairs stored in the IL unit places a ceiling on the level of OL recall. However, Petrich's (1975) model holds that any feature of the list materials or experimental context which is unique to OL, actively processed at acquisition, and available to the learner at recall, may serve as a retrieval cue for the OL unit containing all the $\mathrm{OL}$ pairs.

Response-set interference theory (Postman \& Stark, 1969; Postman, Stark, \& Fraser, 1968) also postulates that the first list becomes inaccessible during secondlist learning. As Petrich (1975) notes, however, there are differences "in the conceptualization of what is suppressed and how" (p. 72). Postman and Stark (1969)

This research was funded by Grant GB-28682, awarded by the National Science Foundation to Dr. Judith A. Petrich. The experiment was conducted in partial fulfillment of the requirements for the author's Master's degree. The author wishes to extend special thanks to Dr. Petrich for her support and guidance, and to Dr. James Pellegrino and Dr. James Voss for their helpful suggestions concerning the manuscript. Requests for reprints should be sent to Harry L. Chiesi, Department of Psychology, University of Pittsburgh, Pittsburgh, Pennsylvania 15260 . propose that during OL subjects restrict their responses to items appearing in the list by developing "selection criteria" or a "response set" based on frequency and relative recency, as well as semantic and formal attributes of the responses. However, Postman and Stark go on to say, "When the required responses are changed in the transfer task, new criteria of selective arousal are established" (p. 176). Furthermore, to the extent that selection criteria are fallible, and intrusions occur, "the criteria of selection must become more restrictive ... The outcome is functionally equivalent to the suppression of the first-list repertoire" (Postman \& Stark, 1969, p. 176). Finally, the inertia of the IL response set prevents the subject from reinstating the OL response repertoire on an immediate retention test.

In addition to their common focus on availability, both theories can accommodate extant data on the effects of distinctive list features upon RI. In typical situations where both the OL and IL response sets are comprised of unrelated adjectives, both theories can explain the observed RI; Petrich, due to the absence of an OL retrieval cue; Postman, due to fallible IL selection criteria, which permit suppression-producing intrusions. In situations where $\mathrm{OL}$ and IL responses are drawn from two distinct form classes (Postman, Keppel, \& Stark, 1965) or two distinct taxonomic categories (Shulman \& Martin, 1970), both theories can explain the elimination of significant RI. Petrich could attribute the effect to the presence of a unique form class- or category-name retrieval cue for the OL unit; Postman, to the lowered probability of intrusions due to the operation of either a category-exclusion or category-inclusion rule for IL selection criteria. To give an example of these rules, consider the participants in the Shulman and Martin (1970) experiment who learned an OL in which all responses were instances of the categnry "professions" and an IL in which all responses were instances of the 
sategory "vegetables." These subjects could minimize intrusions on IL test trials by adopting an IL selection criterion to either (a) give no "profession" responses (the category-exclusion rule) or (b) give only "vegetable" responses (the category-inclusion rule). Since subjects could conceivably have employed either rule, the above data provide no information about the effects of the locus (OL or IL) of distinctive list features upon RI. Both experiments (Postman, Keppel, \& Stark, 1965; Shulman \& Martin, 1970) simply involve comparisons of conditions in which first- and second-list responses are drawn from the same vs. different form class or category-situations where both OL and IL possess a distinctive feature in the different condition.

Interestingly, Petrich's (1975) model entails predictions which are highly divergent from those of responseset theory (Postman \& Stark, 1969) as to whether RI will vary with the locus of distinctive list features like response-set categorization. Response-set theory offers no rationale for maintaining that the locus of a distinctive list feature will affect RI. With OL categorization, a category exclusion rule for IL selection criteria can operate to reduce suppression-producing intrusions; with IL categorization, a category-inclusion rule can perform that same function. Thus, response-set interference theory (Postman \& Stark, 1969) simply maintains that intrusions and RI will be reduced whenever the $\mathrm{OL}$ and IL response sets are highly discriminable. According to Petrich's (1975) model, categorization of the OL response set is necessary for reducing RI. With OL categorization, subjects have a potential category-name retrieval cue for OL; with IL categorization, the occurrence of intrusions and their subsequent storage in the IL unit will be reduced, therefore increasing RI.

A test of the two theories was performed by manipulating the presence (1) or absence (0) of categorized response sets in both OL and IL, producing the following OL-IL conditions: $0-0,0-1,1-0$, and $1-1$. RI was assessed by comparing recall for these experimental groups to that of two control groups, who learned either a categorized first list (C-1) or a noncategorized first list (C-0) prior to solving math problems during IL. The possibility that OL recall would differ for controls learning a categorized first list and those learning a noncategorized first list required that both groups be included in the experiment.

According to response-set theory (Postman \& Stark, 1969) the rank order of the experimental groups in degree of RI should be: $0-0>0-1=1-0>1-1$, since list discrimination cues in the last three groups should reduce intrusions and the resulting RI. It is assumed by the author that Postman would consider two discriminative cues (1-1) to be more effective than a single cue $(0-1,1-0)$ in reducing RI. On the basis of Petrich's (1975) retrieval model, the groups should rank: $0-1>0-0>1-0=1-1$. To elaborate, the presence of a retrieval cue in $1-1$ and $1-0$ should reduce RI signifi- cantly, while $0-1$ should produce more RI than 0-0 since the IL category in the former condition should reduce the storage of $\mathrm{OL}$ responses in the IL unit.

The critical comparison involves the degree of RI obtained in $0-1$ relative to that in 1-0. Postman's theory implies equivalence of the two groups, while Petrich's model suggests that $0-1$ will produce significantly more RI than $1-0$.

\section{METHOD}

\section{Design}

The basic design was a 2 by 2 factorial which varied (a) the presence or absence of a categorized response set in $\mathrm{OL}$ and (b) the presence or absence of such a set in IL. In addition to the four experimental groups, one control group learned a categorized $\mathrm{OL}$, while a second control learned a noncategorized OL. Both control groups solved mathematical progression problems for 9 min (the amount of time spent by subjects in the experimental conditions in learning (L) as their interpolated task.

\section{Materials}

Each list consisted of 10 CVC-noun pairs. The CVC trigrams ranged from 25 to 58 in scaled meaningfulness (Archer, 1960). Formal similarity among trigrams was minimized by using each vowel twice and any consonant only once in the construction of the 10 trigrams. Formal and acoustic similarity between each CVC stimulus and its response was avoided in all lists. The same 10 trigrams appeared in all lists.

Eight 10-item response sets of one- and two-syllable nouns were employed. Mean imagery value per response was 5.69 in the categorized lists and 5.45 in the noncategorized lists (The Colorado Concreteness \& Imagery Norms, 1973). These figures represent the mean for responses with tabled values. For items not tabled in the Colorado norms, values available from the Paivio, Yuille, and Madigan (1968) norms were used. Mean frequency value per response was 46.13 per million in the.categorized lists and 37.10 in the noncategorized lists (Kučera \& Francis, 1967).

The categories represented in the categorized response sets ("natural earth formations," "parts of the body," "means of transportation") were chosen from the Battig and Montague (1969) norms, with the exception of the category "things women wear," which was derived by combining instances of "articles of clothing" and "jewelry." Use of the derived category was necessitated by a simultaneous manipulation of formal/ acoustic similarity in four cells not relevant to the theoretical concerns of this paper.

The lists were counterbalanced so that each list appeared in $\mathrm{OL}$ and IL in all conditions where its categorical and formal/ acoustic properties permitted. Each list appeared with every other list (that was not formally/acoustically similar) at least once in the present design.

\section{Procedure}

Each subject learned OL by the study-test method to an $8 / 10$ criterion. It was hoped that the partial criterion would prevent ceiling effects and thus enable percent recall scores to render a more meaningful measure of $O L$ retention. Visual presentation of the pairs via Carousel projector took place at a $2: 2$-sec rate, with alternating intertrial intervals of 6 and $8 \mathrm{sec}$. Three random orders of the pairs (for study trials) and of the stimuli (for test trials) were used.

For all conditions in which categorized lists were presented, subjects were told twice (immediately prior to learning the list), "One rule that will help you learn the list is that all the responses are examples of [the category]. Please keep this rule in mind while trying to learn the pairs." In addition, all subjects were given standard PA instructions. 
Table 1

Critical Learning and Retention Measures

\begin{tabular}{|c|c|c|c|c|c|c|c|}
\hline & $0-0$ & $0-1$ & 1.0 & $1-1$ & $\mathrm{C}-0$ & $C-1$ & $S E_{p}$ \\
\hline $\begin{array}{l}\text { Trials to Learn } \mathrm{OL} \\
\text { Correct Responses }\end{array}$ & 3.94 & 4.44 & 5.63 & 4.50 & 4.56 & 4.88 & 2.32 \\
\hline $\begin{array}{l}\text { Criterial Trial } \\
\text { Total IL Correct }\end{array}$ & 8.38 & 8.63 & 8.50 & 8.69 & 8.50 & 8.88 & .71 \\
\hline $\begin{array}{l}\text { Correct Responses } \\
\text { Percent OL Recall } \\
\text { RI Scores }\end{array}$ & $\begin{array}{l}61.19 \\
57.06 \\
58.30\end{array}$ & $\begin{array}{l}57.31 \\
43.56 \\
44.51\end{array}$ & $\begin{array}{l}50.31 \\
66.12 \\
66.33\end{array}$ & $\begin{array}{l}59.13 \\
60.25 \\
60.44\end{array}$ & 97.88 & 99.69 & $\begin{array}{l}13.32 \\
21.15\end{array}$ \\
\hline $\begin{array}{l}\text { Correct Responses } \\
\text { Associative Matching }\end{array}$ & 7.50 & 8.13 & 8.13 & 7.38 & 8.75 & 9.13 & 2.04 \\
\hline
\end{tabular}

Immediately following eight IL study-test trials, an unpaced cued recall test for $O L$ retention was given. An associative matching test for OL pairs followed. Subjects were given a sheet of paper listing all the OL stimuli and responses and were asked to match each CVC stimulus with the response it was paired with during $\mathrm{OL}$.

\begin{abstract}
Subjects
The subjects were 96 introductory psychology students at the University of Pittsburgh who were naive to RI experiments and participated in the experiment for partial fulfillment of a course requirement. Sixteen subjects were assigned to each of the six conditions via a blocked random order, so that each condition contained $n$ subjects before the $n+1$ subject was added to any condition.
\end{abstract}

\section{RESULTS}

Unless otherwise noted, a one-way analysis of variance was performed on each measure for which all six groups provided relevant data. The between-groups sum of squares was subsequently partitioned among five orthogonal contrasts. Three contrasts include those inherent in the nested 2 by 2 design which defines the experimental conditions. The two remaining comparisons involve experimental vs. control and categorized (C-1) vs. noncategorized (C-0) control. In all analyses, the the minimum significance level was $\mathrm{p}<.05$.

Means for all analyzed response measures are presented in Table 1. The standard error $\left(\mathrm{SE}_{\mathrm{p}}\right)$ reported for each measure represents a pooled estimate for all the means calculated from the mean square within.

\section{List 1 Learning}

The overall $\mathrm{F}$ test and the five orthogonal contrasts (including C-1 vs. C-0) failed to reveal any significant differences in List 1 trials to criterion (see Table 1 for means). These results suggest that the groups were approximately equivalent in PA leaming ability and, more importantly, that there were no significant differences in the rate of learning categorized lists (5.01 trials) vs. noncategorized lists (4.31 trials). This conclusion is supported by the analysis of correct responses on the criterial OL trial, which also failed to yield any significant effects from the five orthogonal contrasts. Students learning a categorized OL produced an average of 8.63 correct responses on the criterial OL trial; those learning a noncategorized OL produced 8.45 .

\section{List 2 Learning}

A 2 by 2 analysis (OL list type by IL list type) o: total List 2 correct responses demonstrated a significan OL by IL interaction $[F(1,60)=3.82, p<.05]$. The effect shows that subjects learning a different type 01 list during IL (the $0-1$ and 1-0 conditions) give fewer correct responses (53.82) than subjects who learn the same type of list (0-0 and 1-1) in both stages (60.16)

\section{OL Intrusions During IL}

Among the four experimental conditions, only twc interlist intrusions were made during $\mathrm{IL}-$ both of then by a single subject in the $0-0$ condition.

\section{List 1 Percent Recall}

A percent OL recall score was calculated for eack. subject by dividing the number correct on the cuec recall test by the number correct on the criterial $\mathrm{OI}$ trial. An arc-sine transformation was performed on each. percentage score prior to the analysis.

The $\mathrm{C}-1$ vs. $\mathrm{C}-0$ comparison revealed that the twc control groups did not differ significantly $[F(1,90)<1]$ on OL retention. Since the percent $\mathrm{OL}$ recall means fos C-1 (99.69\%) and C-0 (97.88\%) were virtually identical it follows that percent OL recall scores for the experi. mental groups would be virtually identical to RI scores obtained by dividing recall scores in each experimenta: group by the recall mean of the appropriate contro group, as shown in Table 1. Therefore, it was legitimate to use percent $\mathrm{OL}$ recall scores as a measure of RI for the experimental groups in the following analyses

Further examination of the data revealed a significant $\mathrm{OL}$ category effect $[F(1,90)=5.36, p<.05]$, as less RI (or higher OL recall) was exhibited by subjects learning a categorized first list $(63.18 \%)$ than those learning a noncategorized first list $(50.31 \%)$. The sig. nificant IL category effect $[F(1,90)=4.49, p<.05]$ demonstrated that more RI was exhibited by subjects learning a categorized second list $(51.91 \%)$ than by those learning a noncategorized second list (61.59\%). As expected, average recall for the two control groups $(98.78 \%)$ was much better $[\mathrm{F}(1,90)=113.89, \mathrm{p}<.001]$ than that of the experimental groups $(56.72 \%)$. Elimi nating the control groups and performing a 2 by 2 (OL list type by IL list type) analysis of variance preserved the OL effect $[F(1,60)=4.65, p<.05]$, and 
reduced the IL effect to marginal significance $[F(1,60)=$ $3.90, \mathrm{p}<.06]$.

According to Petrich's (1975) model, the experimental groups should rank 0-1 $>0-0>1-0=1-1$ in degree of RI. The actual means corresponding to this predicted ranking were $43.56 \%>57.06 \%>66.12 \% \cong 60.25 \%$, providing rather close agreement with the model. Post-hoc $F$ tests revealed that $0-1$ produced significantly greater RI than $0.0[F(1,90)=4.61, p<.05]$; that the $0-0$ vs. 1.0 difference was not statistically reliable $[F(1,90)<1]$, though in the expected direction; and that the slight and unexpected superiority of $1-0$ over $1-1$ failed to approach significance $[\mathrm{F}(1,90)<1]$. Petrich's model also predicts that $0-1$ should produce significantly greater RI than 1.0 . The obtained difference $(22.56 \%)$ in this critical comparison was in the predicted direction and highly significant $[F(1,90)=9.83, p<.01]$.

\section{Intrusions at Recall}

Only one interlist intrusion was made at recall, by a subject in the $1-0$ condition.

\section{Associative Matching}

Analysis of matching scores yielded a significant $F$ value only for the experimental vs. control comparison $[F(1,90)=7.17, p<.01]$, as subjects in the experimental conditions matched an average of 7.59 pairs correctly, while control subjects matched 8.94 pairs correctly.

\section{DISCUSSION}

The experiment was designed to test the predictions of Petrich's (1975) retrieval model against those of Postman and Stark's (1969) response-set theory as to whether RI would vary with the locus of categorized response sets. To reiterate, Petrich's model implies that only OL categorization will provide the retrieval cue necessary to reduce RI; thus, 1-0 should produce significantly less RI than $0-1$. Postman's theory suggests that either OL or IL categorization will reduce the probability of suppression-producing intrusions; thus, 1-0 and $0-1$ should produce equivalent amounts of RI.

Contrary to the latter predictions, RI does vary with the locus of categorized response sets: OL categorization significantly reduced RI, IL categorization significantly increased $\mathrm{RI}$, and 1.0 produced significantly less (22.56\%) RI than 0-1. In attempting to show how response-set theory could account for these results, one might argue that IL categorization per se leads to $\mathrm{OL}$ suppression. This ad-hoc assumption absolves intrusions from any responsibility for RI in $0-1$, although the formal statements of response-set theory (Postman \& Stark, 1969; Postman, Stark, \& Fraser, 1968) attribute RI to suppression produced by the intrusions which typically occur under 0-0 conditions. If one adopts a hybrid theory containing two suppression mechanisms (one activated by common properties among IL responses, the other by OL intrusions during IL), it then becomes difficult to account for RI data easily explained by the present form of response-set theory. For example, if the presence of common properties among IL responses produces OL suppression, how does one explain the fact that subjects learning two lists whose responses are drawn from two distinct form classes (e.g., adjectives vs. numbers) show less RI than subjects learning two lists in which the responses are drawn from the same form class (Petrich, 1975, Experiment 3), when both groups of subjects learn the same IL? A second argument might be made that the recall differences obtained in the present experiment were products of negative transfer, which typically accompanies RI. However, since the condition (1-0) producing the highest $\mathrm{OL}$ recall also demonstrated the poorest performance in second-list learning, such an explanation is hardly credible. Finally, one should note that both of the above arguments fail to deal with the fact that $\mathrm{OL}$ intrusions (not IL response properties or negative transfer) are responsible for activating the suppression mechanism proposed by response-set theory. In order to accommodate the present findings, Postman and Stark's (1969) response-set theory would seem to require major revisions which provide a rationale for maintaining that (a) high specificity of OL selection criteria is a more important facter than the ability to quickly form new IL criteria, and (b) the rapid development of highly specific IL criteria (with IL categorization) may increase RI.

While the results provide little evidence in support of the current version of Postman's theory, they can be readily incorporated within the framework of Petrich's model-without the help of any additional assumptions. First of all, the facilitative effect of OL categorization concurs with Petrich's contention that a retrieval cue for the OL memory unit is the key to reducing RI. Second, the inhibitory effect of IL categorization upon recall and the marked inferiority of $0-1$ to $0-0$ are consistent with the prediction that variables reducing the storage of $\mathrm{OL}$ intrusions in the IL unit will increase the degree of RI-provided, of course, that no OL retrieval cue is available. Third, the actual rankings of the experimental groups in degree of RI corresponded rather closely to the rankings predicted by the model. Finally, the failure to find an effect of $\mathrm{OL}$ categorization on associative matching indicates that students in all the experimental conditions had approximately the same number of associations intact in the OL memory unit-and that the superior recall of those learning categorized lists was a result of the ability to recall pairs from the OL (rather than just the IL) memory unit.

With respect to the paucity of interlist intrusions during IL, it is critical to note that, while Petrich's (1975) model is based on her findings concerning 
the probability of recalling OL responses (on a final retention test) which intruded during $I L$, those findings were obtained under a procedure which differed radically from that used in the present study. Petrich's (1975) procedure involved subject-paced test trials and instructions to recall the correct response and any wrong responses (intrusions) that came to mind upon viewing the stimulus. In the present experiment, subjects were given standard 2-sec test trials and instructions to recall only the correct response. Inasmuch as Petrich's (1975) procedure was designed to make overt those intrusion processes which remain largely covert under standard 2 -sec test trials, the intrusion data obtained in this experiment are not comparable in theoretical relevance to those obtained by Petrich.

While less directly relevant to the theoretical controversy addressed by this investigation, the inability of students with a categorized OL to recall all pairs correctly given on the criterial OL trial bears further mention. It is presently assumed that output interference from the dominant IL unit (Postman, Stark, \& Henschel, 1969) hinders the retrieval of some OL Pairs (Petrich, 1975). The output interference phenomenon, an instance of what Runquist (1975) has termed trace interaction, may also account for the slight superiority of control subjects on the associative matching test, since controls did not learn a second list which could provide such interference.

To conclude, the present investigation provides evidence in favor of Petrich's (1975) retrieval model, while posing explanatory problems for Postman and Stark's (1969) response-set interference theory. At minimum, the data require the modification of responseset theory by corollaries that explain why (a) OL is the critical locus for categorization with respect to reducing $\mathrm{RI}$ and (b) IL categorization may actually increase RI.

\section{REFERENCE NOTE}

1. The Colorado concreteness and imageny norms. Program on Cognitive Factors in Human Learning and Memory. Boulder. Colorado: Institute for the Study of Intellectual Behavior, University of Colorado, 1973.

\section{REFERENCES}

ArCher, E. A reevaluation of the meaningfulness of all possible CVC trigrams. Psychological Monographs, 1960, 74, 10.

Battig, W. F., \& Montague, W. E. Category norms for verbal items in 56 categories: A replication and extension of the Connecticut category norms. Journal of Experimental Psychology Monograph, 1969. 80(3. Pt. 2), 1-45.

Kučra, H., \& Francis, W. N. Computational analysis of present-day American English. Providence, R.I: Brown University Press, 1967.

Paivio, A., Yuille, J. C., \& Madigan, S. Concreteness, imagery and meaningfulness values for 925 nouns. Journal of Experimental Psychology, 1968, 76(1, Pt. 2).

Petrich, J. A. Storage and retrieval processes in unlearning. Memory \& Cognition, 1975, 3, 63-74.

Postman, L., Keppel, G., \& Stark, K. Unlearning as a function of the relationship between successive response classes. Joumal of Experimental Psychology, 1965, 69, 111-118.

Postman, L., \& StaRk, K. Role of response availability in transfer and interference. Joumal of Experimental Psychology, 1969, 79, 168-177.

Postman, L., Stark, K., \& Fraser, J. Temporal changes in interference. Journal of Verbal Learning and Verbal Behavior, 1968, 7, 672-694.

Postman, L., Stark, K., \& Henschel, D. Conditions of recovery after unlearning. Journal of Experimental Psychology, 1969, 82, 1. Pt. 2.

Runquist, W. N. Interference among memory traces. Memory \& Cognition, 1975, 3, 143-159.

Shulman, H. G., \& Martin, E. Effects of response-set similarity on unlearning and spontaneous recovery. Journal of Experimental Psychology, 1970, 86, 230-235.

(Received for publication August 8, 1975; revision accepted December $15,1975$. ) 